\title{
Submillimeter water and ammonia absorption by the peculiar $z \approx 0.89$ interstellar medium in the gravitational lens of the PKS 1830-211 system
}

\author{
K. M. Menten ${ }^{1}$, R. Güsten ${ }^{1}$, S. Leurini ${ }^{2}$, S. Thorwirth ${ }^{1}$, C. Henkel $^{1}$, B. Klein ${ }^{1}$, C. L. Carilli ${ }^{3}$, and M. J. Reid ${ }^{4}$ \\ 1 Max-Planck-Institut für Radioastronomie, Auf dem Hügel 69, 53121 Bonn, Germany \\ e-mail: [kmenten;rguesten; sthorwirth; chenkel;bklein]@mpifr-bonn.mpg.de \\ 2 ESO, Karl-Schwarzschild-Strasse 2, 85748 Garching bei München, Germany \\ e-mail: sleurini@eso.org \\ 3 National Radio Astronomy Observatory, PO Box 0, Socorro, NM 87801-0387, USA \\ e-mail: ccarilli@nrao.edu \\ ${ }^{4}$ Harvard-Smithsonian Center for Astrophysics 60 Garden Street Cambridge, MA 02138, USA \\ e-mail: reid@cfa.harvard.edu
}

Received 24 April 2008 / Accepted 15 October 2008

\section{ABSTRACT}

\begin{abstract}
Using the Atacama Pathfinder Experiment (APEX) telescope, we have detected the rotational ground-state transitions of orthoammonia and ortho-water toward the redshift $\approx 0.89$ absorbing galaxy in the PKS 1830-211 gravitational lens system. We discuss our observations in the context of recent space-borne data obtained for these lines with the SWAS and Odin satellites toward Galactic sources. We find commonalities, but also significant differences between the interstellar media in a galaxy at intermediate redshift and in the Milky Way. Future high-quality observations of the ground-state ammonia transition in PKS 1830-211, together with inversion line data, will lead to strong constraints on the variation in the proton to electron mass ratio over the past 7.2 Gyr.
\end{abstract}

Key words. cosmology: observations - gravitational lensing - astrochemistry - ISM: abundances - ISM: molecules

\section{Introduction}

The gravitational lens system involving the bright compact radio and millimeter source PKS 1830-211 is remarkable for several reasons: a distant quasi-stellar object (QSO) at a redshift, $z$, of 2.507 (Lidman et al. 1999) is imaged into two strong, milliarcsecond (mas) size sources separated by $\approx 970$ mas, corresponding to $7.3 \mathrm{kpc}$ at $z=0.89^{1}$ (Jin et al. 2003). These components appear embedded in extended emission, part of which, at high resolution, presents an Einstein ring (Subrahmanyan et al. 1990; Jauncey et al. 1991).

Through an act of "cosmic conspiracy", the line of sight to the background QSO passes through or near several galaxies (Meylan et al. 2005) and, placed at Galactic longitude +12.2 and latitude -5.7 , the bulge of the Milky Way, causing local neutral hydrogen (HI) absorption (Subrahmanyan et al. 1992). Courbin et al. (2002) give a historical summary of this complex picture. Most importantly, the line of sight passes through a spiral galaxy at a redshift of $\approx 0.89$ (Winn et al. 2002), in which continuum emission from the QSO is absorbed in lines of atomic hydrogen and hydroxyl (OH) (Chengalur et al. 1999; Koopmans \& de Bruyn 2005) and transitions from a variety of molecules in the interstellar media of two different spiral arms (Wiklind \& Combes 1996, 1998). The much deeper absorption has its centroid at $z=0.88582$ (zero velocity in the heliocentric frame) and is toward the south-western (SW) image

1 Throughout this paper we are using a $\Lambda$ cosmology with $H_{0}=$ $73 \mathrm{~km} \mathrm{~s}^{-1} \mathrm{Mpc}^{-1}, \Omega_{\mathrm{M}}=0.28$, and $\Omega_{\Lambda}=0.72$ (Spergel et al. 2007). 1 arcsec corresponds to $7.54 \mathrm{kpc}$.
(Frye et al. 1997; Muller et al. 2006), which is rendered undetectable at optical wavelengths by the dust mixed with the molecular material (Winn et al. 2002). The absorption appears to be caused by material in a spiral arm of the lensing galaxy at projected distance of $\approx 0$ '. 4 from the galaxy's center $(3 \mathrm{kpc})$, clearly defined in the $I$-image presented by these authors. We note that in the Milky Way the bulk of the molecular gas is distributed between Galactocentric radii of 4 and $7 \mathrm{kpc}$ (Bronfman et al. 1988).

Wiklind \& Combes (1998) detected a second, weaker absorption cloud with a velocity shifted by $-147 \mathrm{~km} \mathrm{~s}^{-1}(z=$ 0.88489 ) relative to the primary absorption and toward the NE image (Muller et al. 2006), whose location is displaced by $0 . ' 59$ $(4.4 \mathrm{kpc})$ from galaxy's center.

For completeness, we mention that there is another HI absorber at $z=0.19$ that absorbs the NE and, weaker, the SW image as well as part of PKS 1830-211's extended emission (Lovell et al. 1996). The nature of this low opacity $\left(\tau_{\mathrm{HI}} \sim 0.01\right)$ system's host is uncertain but possibly we are dealing with another intervening spiral galaxy of low surface brightness. No molecular absorption has been detected at this redshift (Wiklind \& Combes 1998).

Wiklind and Combes detected absorption in $\mathrm{CO}$ and in a number of molecules with (much) higher dipole moments, namely $\mathrm{HCN}, \mathrm{HCO}^{+}, \mathrm{CS}$, and $\mathrm{N}_{2} \mathrm{H}^{+}$(Wiklind \& Combes 1996 , 1998). Menten et al. (1999) added $\mathrm{C}_{2} \mathrm{H}, \mathrm{HC}_{3} \mathrm{~N}$, and $\mathrm{C}_{3} \mathrm{H}_{2}$ to this list. A number of rare isotopologues of $\mathrm{C}-, \mathrm{N}-, \mathrm{O}-$, and S-bearing species were imaged by Muller et al. (2006), who found isotopic ratios differing from Milky Way values. Menten et al. (1999) 
compared PKS 1830-211's molecular make-up with Galactic dark clouds and found many similarities. Recently, Henkel et al. (2008) observed a total of ten metastable inversion transitions of ammonia $\left(\mathrm{NH}_{3}\right)$ and found the observations to be consistent with $80-90 \%$ of the ammonia-bearing gas being warm $(\sim 80 \mathrm{~K})$. Amazingly, to explain the intensities of the lines at high energy levels (up to $1000 \mathrm{~K}$ above the ground-state) they have to invoke a hot $>600 \mathrm{~K}$ medium for which in the Milky Way the only known counterpart would be the Sgr B2 region near the Galactic center (Huettemeister et al. 1995; Wilson et al. 2006).

Redshifted absorbers provide the possibility to detect spectral lines that are unobservable from the ground due to attenuation by the Earth's atmosphere, namely low excitation water $\left(\mathrm{H}_{2} \mathrm{O}\right)$ lines that cause the bulk of the terrestrial absorption. This was exploited by Combes \& Wiklind (1997), who discovered absorption in the $1_{10}-1_{01}$ ground-state transition of ortho-water $\left(\mathrm{o}-\mathrm{H}_{2} \mathrm{O}\right)$ toward the "other" rich molecular intermediate-redshift $(z=0.685)$ system B0218+357. The terrestrial, pressure-broadened $1_{10}-1_{01} \mathrm{H}_{2} \mathrm{O}$ line near $557 \mathrm{GHz}$ causes the broadest range of absorption in the Earth's atmosphere below $1 \mathrm{THz}$, rendering the $\pm 50 \mathrm{GHz}$ around it practically impossible to observe from the ground, even from the excellent $5100 \mathrm{~m}$ high site of the APEX telescope. Considerable effort was put in the investigation of this line in the Milky Way; it was a main motivation for two space missions, the Submillimeter Wave Astronomy Satellite (SWAS Melnick et al. 2000) and Odin (Nordh et al. 2003; Hjalmarson et al. 2003). Among many other things, Odin also delivered the first astronomical detection of the $1_{0}-0_{0}$ ground-state transition of ammonia, $\mathrm{NH}_{3}$, which near $572 \mathrm{GHz}$ is also unobservable from the ground due to absorption from the $557 \mathrm{GHz} \mathrm{H}_{2} \mathrm{O}$ line's broad line wings.

Here we report the detection of the $\mathrm{H}_{2} \mathrm{O}$ and the $\mathrm{NH}_{3}$ orthoground-state rotational lines toward PKS 1830-211 with the APEX telescope ${ }^{2}$. In Sect. 2 we describe the observations and data reduction. Our results are presented in Sect. 3 and their analysis in Sect. 4. Astrochemical implications of our study are discussed in Sect. 5.

\section{Observations and data reduction}

Our observations were made in 2007 July 2-5 under generally good weather conditions with APEX, the 12-m Atacama Pathfinder Experiment telescope (Güsten et al. 2006). Opacities at the observing frequencies $(\approx 300 \mathrm{GHz})$ were between 0.02 and 0.12 throughout the observations and (single sideband) system temperatures between 240 and $390 \mathrm{~K}$. We observed the ortho-ground state lines of water $\left(\mathrm{H}_{2} \mathrm{O}\right)$ and ammonia $\left(\mathrm{NH}_{3}\right)$, namely the $\mathrm{H}_{2} \mathrm{O} J_{K_{a} K_{c}}=1_{10}-1_{01}$ and the $\mathrm{NH}_{3} J_{K}=1_{0}-0_{0}$ transitions. These were redshifted from their rest frequencies of $556936.00 \pm 0.05$ and $572498.1 \pm 0.3 \mathrm{MHz}$ to observing frequencies, $v$, of 295328.3 and 303580.4 and $\mathrm{MHz}$, respectively. We assumed the redshift accurately determined from mm-/cmwavelength absorption measurements, namely $z=0.88582$. All spectra are presented with velocities in the heliocentric frame; add $12.1 \mathrm{~km} \mathrm{~s}^{-1}$ for LSR velocities.

Both lines were measured in the lower sideband (LSB) with the APEX 2a facility receiver (Risacher et al. 2006). Calibration was obtained using the chopper wheel technique, considering the different atmospheric opacities in the signal and image sidebands of the employed double sideband receivers. To ensure flat spectral baselines, the newly available wobbling secondary was

\footnotetext{
2 APEX observations of the $\mathrm{H}_{2} \mathrm{O}$ line toward PKS 1830-211 have been announced previously by Wiklind \& Combes (2005).
}

chopped with a frequency of $1.5 \mathrm{~Hz}$ and a throw of $100^{\prime \prime}$ about the cross elevation axis. The wobbler was operated in symmetric mode, which means that source and off position are interchanged between subsequent subscans, which cancels any asymmetries in the optical paths. Such observations deliver a reliable estimate of the continuum level. The radiation was analyzed with the MPIfR Fast Fourier Transform spectrometer, which provides 16384 frequency channels over the $1 \mathrm{GHz}$ intermediate frequency bandwidth (Klein et al. 2006). To increase the signal to noise ratio, the spectra were smoothed to effective velocity resolutions appropriate for the measured linewidths, i.e., 4.0 and $3.9 \mathrm{~km} \mathrm{~s}^{-1}$ for the $\mathrm{H}_{2} \mathrm{O}$ and $\mathrm{NH}_{3}$ lines, respectively. To check the telescope pointing, drift scans were made across the strong continuum sources Sgr B2 N, G10.47+0.03, Jupiter, and Mars. Pointing corrections were derived from these measurements. The pointing was found to be accurate to within $\approx 3^{\prime \prime}$, acceptable given the $F W H M$ beam size, $\theta_{\mathrm{B}}$, which is $21^{\prime \prime} F W H M$ at $300 \mathrm{GHz}$.

We have converted our line intensities, measured with the chopper wheel technique, into a flux density, $S$, scale (i.e., in Jansky units), assuming the aperture efficiency observationally determined by Güsten et al. (2006) at $352 \mathrm{GHz}$ and higher frequencies extrapolated to $300 \mathrm{GHz} ; 1 \mathrm{~K}$ of antenna temperature corrected for above the atmosphere corresponds to $37.7 \mathrm{Jy}$. We present our observed spectra in Fig. 1 with the continuum levels indicated (see Sect. 3.1). No baselines were subtracted from these spectra.

\section{Results}

\subsection{Continuum intensities}

In the double-sideband APEX 2 a receiver, the two sidebands are $12 \mathrm{GHz}$ apart and continuum emission from both sidebands is detected. The effective center frequencies for the determination of the continuum levels are 301.3 and $309.6 \mathrm{GHz}$, respectively, for the two sidebands. We determine continuum levels, $S_{\mathrm{C}}$, of $1.73 \mathrm{Jy}$ at the former and $1.65 \mathrm{Jy}$ at the latter frequency, respectively. Given the absolute calibration uncertainties, which we estimate at $10 \%$, the difference between the two measurements is not significant. Keeping in mind that PKS 1830-211 is highly variable, we note that our flux densities are higher than the 1.3 Jy measured with the Large APEX Bolometer Camera (LABOCA) shortly before our observations, i.e., in 2007 May (Weiss and Schuller, personal communication). The LABOCA's effective (filter-averaged) frequency is $345 \mathrm{GHz}, 49 \mathrm{GHz}$ higher than the $\mathrm{H}_{2} \mathrm{O}$ line frequency. If PKS 1830-211's submillimeter flux did not vary between then and the time of our measurements, comparison of our and the LABOCA data shows that the trend of dropping flux with increasing frequency continues at least up to $345 \mathrm{GHz}$.

We estimate the uncertainties of our absolute flux density calibration and the systematic uncertainties in the optical depth determinations (see Sect. 3.3) to be $\approx 10 \%$. This means that the uncertainties in the optical depth determinations are dominated by the limited signal-to-noise ratio of the line measurements.

\subsection{The magnification ratio and covering factor}

With our $\approx 20^{\prime \prime} F W H M$ beam we are detecting the sum of the continuum flux densities from the NE and the SW images. However, from interferometric imaging it is clear that observed absorption at zero velocity (relative to $z=0.88582$ ) only arises toward the weaker SW image (Frye et al. 1997; Menten et al. 1999; Muller et al. 2006). Thus, we must correct the observed apparent optical depths to true optical depths taking this into 


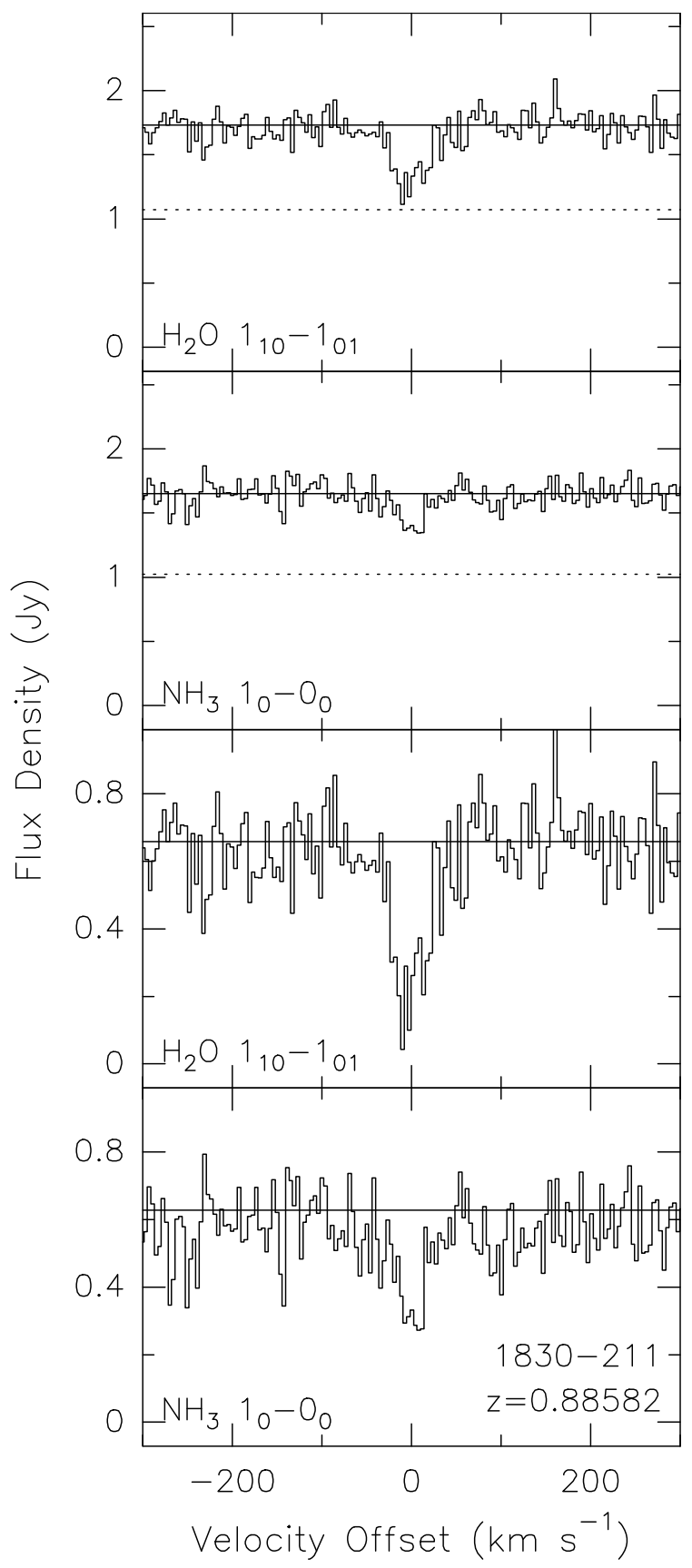

Fig. 1. Spectra of absorption in the $J_{K_{a} K_{c}}=1_{10}-1_{01}$ transition of water vapor and the $J_{K}=1_{0}-0_{0}$ transition of ammonia taken with the APEX telescope toward PKS 1830-211. The velocity scale is in the heliocentric frame and assumes a redshift of 0.88582 . For the two upper spectra, the continuous horizontal line gives the continuum level discussed in Sect. 2 while the difference between the dotted and continuous lines gives the actually absorbed continuum level from the SW component (total covering factor 0.38; see Sect. 3.3). For clarity, in the lower panels the $\mathrm{H}_{2} \mathrm{O}$ and $\mathrm{NH}_{3}$ spectra are shown relative to the actually absorbed continuum level.

account. We here define the "magnification ratio", $\Re$, for PKS 1830-211 as the ratio of the radio or (sub)millimeter flux densities of the stronger NE to the weaker SW image. Due to time delay between the two ray paths, this ratio is temporally variable, but if one accounts for that, $\Re \approx 1.5$ has been estimated in the radio regime (Lovell et al. 1998). Observations with the IRAM Plateau de Bure Interferometer (PdBI) taken between 1995 and 2003 in the $3 \mathrm{~mm}$ wavelength range show $\mathfrak{R} \approx 1.5-1.75$
(Muller et al. 2006), with a best value of 1.66. The flux density of the SW image is $\eta_{\text {cov }} S_{\mathrm{C}} \equiv S_{\mathrm{C}} /(1+\Re)$. Note that here the "covering factor" $\eta_{\text {cov }}$, which accounts for the fact that only the SW image is absorbed, ranges from 0.36 to 0.40 , corresponding to the range in $\Re$ given above. What fraction of that image's few mas/few pc size area is absorbed is not considered and is assumed to be near unity as suggested by Very Long Baseline Array (VLBA) observations (Carilli et al. 1998).

\subsection{Observed and corrected optical depths}

A Gaussian fit to the $\mathrm{H}_{2} \mathrm{O}$ line yields a peak line signal, $S_{\mathrm{L}}$, of $-0.47(0.11) \mathrm{Jy}$, and an integrated line signal, $\int S_{\mathrm{L}} \mathrm{d} v$, of $-22.1(2.0) \mathrm{Jy} \mathrm{km} \mathrm{s}^{-1}$, an $F W H M$ linewidth, $\Delta v$, of $44(5) \mathrm{km} \mathrm{s}^{-1}$ and velocity offset, $v$, of $-1.8(2.0) \mathrm{km} \mathrm{s}^{-1}$ relative to $z=$ 0.88582 . Using this line and the continuum flux density derived in Sect. 3.1 (10\% uncertainty for the latter), we calculate an apparent optical depth,

$\tau_{\text {app }}=-\ln \left(1-\left|S_{\mathrm{L}}\right| / S_{\mathrm{C}}\right)$

of $0.32_{-11}^{+15}$ and a velocity-integrated apparent optical depth, $\int \tau_{\text {app }} \mathrm{d} v$ of $15.0 \mathrm{~km} \mathrm{~s}^{-1}$.

For the $\mathrm{NH}_{3}$ line we find $S_{\mathrm{L}}=-0.29(6) \mathrm{Jy}, \int S_{\mathrm{L}} \mathrm{d} v=$ $-10.4(1.6) \mathrm{Jy} \mathrm{km} \mathrm{s}^{-1}, \Delta v=34$ (7) $\mathrm{km} \mathrm{s}^{-1}$, and $v=$ $0.5(2.5) \mathrm{km} \mathrm{s}^{-1}$, yielding $\tau_{\text {app }}=0.19_{-0.06}^{+0.08}$ and $\int \tau_{\text {app }} \mathrm{d} v=$ $6.4 \mathrm{~km} \mathrm{~s}^{-1}$.

Replacing $S_{\mathrm{C}}$ with $\eta_{\text {cov }} S_{\mathrm{C}}$ in the above equation and considering that $0.36<\eta_{\text {cov }}<0.40$, we obtain, for the $\mathrm{H}_{2} \mathrm{O}$ line, a true optical depth of $1.3_{-0.6}^{+\infty}$ and $61 \mathrm{~km} \mathrm{~s}^{-1}$ for its velocity-integrated value. This means that from our data we cannot give an upper bound for the line's opacity.

For the $\mathrm{NH}_{3}$ line we calculate $0.62_{-0.24}^{+0.46}$ and $22 \mathrm{~km} \mathrm{~s}^{-1}$ for the true optical depth and its velocity-integrated value, respectively.

For a spectral line from an upper level $u$, with energy $E_{u}$, to a lower level $l$, with energy $E_{l}$, the column density in the upper level is

$N_{u}=\frac{8 \pi v^{3}}{c^{3} A_{u l}}\left[\mathrm{e}^{h v / k T_{\mathrm{ex}}}-1\right]^{-1} \int \tau \mathrm{d} v$,

where $T_{\mathrm{ex}}$ is the excitation temperature, $A_{u l}$ the Einstein $A$ coefficient; $h$ and $k$ are the Planck and Boltzmann constants, respectively, and $c$ is the speed of light. $v=\left(E_{u}-E_{l}\right) / h$ is the line's rest frequency. In convenient units,

$$
\begin{aligned}
N_{u}\left(\mathrm{~cm}^{-2}\right)= & 93.28 \frac{v^{3}(\mathrm{GHz})}{A_{u l}\left(\mathrm{~s}^{-1}\right)}\left[\mathrm{e}^{0.048 v(\mathrm{GHz}) / T_{\mathrm{ex}}(\mathrm{K})}-1\right]^{-1} \\
& \times \int \tau \mathrm{d} v\left(\mathrm{~km} \mathrm{~s}^{-1}\right) .
\end{aligned}
$$

The total column density is given by

$N_{\text {rot }}=\frac{N_{u}}{g_{u}} \mathrm{e}^{E_{u} / k T_{\text {rot }}} Q\left(T_{\text {rot }}\right)$,

where $g_{u}$ is the line's upper level degeneracy and $Q$ is the partition function for the rotation temperature, $T_{\text {rot }}$. We assume Local Thermodynamic Equilibrium (LTE), which means $T_{\text {rot }}=T_{\text {ex }}$.

\section{Analysis}

\subsection{Water vapor absorption}

For the $\mathrm{H}_{2} \mathrm{O} J_{K_{a} K_{c}}=1_{10}-1_{01}$ transition, $g_{u}=g_{l}=9$ and $A_{u l}=3.45 \times 10^{-3} \mathrm{~s}^{-1}$, where $u$ and $l$ denote the $1_{10}$ and $1_{01}$ 
energy levels, respectively. To consider the state of thermalization of this line, we examine the rates for collisional deexcitation from the $1_{01}$ level, which are accessible at the website of the Leiden Atomic and Molecular Database (LAMDA) ${ }^{3}$ (Schöier et al. 2005). The calculations of Grosjean et al. (2003) for collisions between ortho- $\mathrm{H}_{2}$ and ortho- $\mathrm{H}_{2} \mathrm{O}$ at low temperatures (5-20 K), extended to higher temperatures (140 K), using the results of Phillips et al. (1996), yield rate coefficients for transitions from the upper $1_{10}$ to the lower $1_{01}$ level, $\gamma_{u l}$, between $8.2 \times 10^{-12}$ and $2.2 \times 10^{-11} \mathrm{~cm}^{3} \mathrm{~s}^{-1}$ for kinetic temperatures between 5 and $80 \mathrm{~K}$, where the latter number applies for the bulk of the gas from which absorption in the ammonia inversion transitions is also observed (Henkel et al. 2008). This implies a critical density, $n_{\text {crit }}=A_{u l} / \gamma_{u l}$, of several times $10^{8} \mathrm{~cm}^{-3}$, which is very much higher than any plausible value of the hydrogen density of the absorbing material. It is therefore straightforward to assume that the $\mathrm{H}_{2} \mathrm{O}$ level populations are thermalized at the temperature of the cosmic microwave background, $T_{\mathrm{CMB}}$. In other words, $T_{\mathrm{ex}}$ in Eq. (3) is equal to $T_{\mathrm{CMB}}=(1+z) 2.728 \mathrm{~K}=$ $5.145 \mathrm{~K}$. Using these values and the lower limit to the integrated optical depth discussed in Sect. 3.3, i.e. $0.7 \times 39=27.3 \mathrm{~km} \mathrm{~s}^{-1}$, with $T_{\mathrm{ex}}=T_{\mathrm{CMB}}$, Eq. (3) gives an upper level column density of $N_{1_{10}}=7.1 \times 10^{11} \mathrm{~cm}^{-2}$.

Noting that the ortho- $\mathrm{H}_{2} \mathrm{O}$ partition function for this temperature, $Q_{\mathrm{o}-\mathrm{H}_{2} \mathrm{O}}(5.145 \mathrm{~K})$, is $\approx 9.05$, we can use Eq. (4) to find that the total ortho- $\mathrm{H}_{2} \mathrm{O}$ column density, $N\left(\mathrm{o}-\mathrm{H}_{2} \mathrm{O}\right)$, is $9.9 \times 10^{13} \mathrm{~cm}^{-2}, 182.14$ times higher than $N_{10}$ and, from detailed balance, that almost all ortho- $\mathrm{H}_{2} \mathrm{O}$ molecules $(99.45 \%)$ are in the $1_{01}$ ground-state level! To verify this, we used the non-LTE radiative transfer program $\mathrm{RADEX}^{4}$ created and made available to the community by van der Tak et al. (2007) to perform statistical equilibrium calculations. For a set of input excitation conditions, namely background temperature $(5.145 \mathrm{~K})$, kinetic temperature, and $\mathrm{H}_{2}$ density, RADEX calculates level populations with column density and line width as radiative transfer input parameters and returns the excitation temperatures and optical depths of user-selected spectral lines.

Running RADEX, we find that for densities up to $10^{6} \mathrm{~cm}^{-3}$ the excitation temperature does not become significantly higher than $T_{\mathrm{CMB}}$. For the line width derived in Sect. 3 RADEX returns the optical depth value discussed above $(\tau=0.7)$ for an ortho$\mathrm{H}_{2} \mathrm{O}$ column density of $9.3 \times 10^{13} \mathrm{~cm}^{-2}$, close to above estimate. This result is independent of all plausible values of the kinetic temperature and densities up to $10^{6} \mathrm{~cm}^{-3}$.

We emphasize that the above estimate is calculated for the lower limit of the true integrated optical depth derived in Sect. 3.3 and a strict lower limit. The upper uncertainty bound of the optical depth is not determinable from our noisy data.

\subsection{Ammonia absorption}

For the $\mathrm{NH}_{3} J_{K}=1_{0}-0_{0}$ transition, $g_{u}=12$ and for the $(J, K)=$ $(1,1)$ transition $g_{u}=6$. These values include factors for nuclear spin degeneracies. The very different Einstein $A$ coefficients for these lines are $A_{1_{0}-0_{0}}=1.61 \times 10^{-3} \mathrm{~s}^{-1}$ and $A_{1,1}=1.71 \times 10^{-7} \mathrm{~s}^{-1}$, respectively.

Deexcitation rates for collisions of $\mathrm{H}_{2}$ with ortho- $\mathrm{NH}_{3}$ have been calculated by Danby et al. (1988) and are also accessible at the LAMDA website. We find that the rate for collisional deexcitation, $\gamma_{01}$, from the $1_{0}$ to the $0_{0}$ level for temperatures between 15 and $300 \mathrm{~K}$ varies little and is between $4.3 \times 10^{-11}$

\footnotetext{
3 http://www.strw.leidenuniv.nl/ moldata/

${ }^{4}$ http://www.strw. leidenuniv.nl/ moldata/radex.html
}

and $6.2 \times 10^{-11} \mathrm{~cm}^{3} \mathrm{~s}^{-1}$. The critical density of the $J_{K}=1_{0}-0_{0}$ transition, $n_{\text {crit }}\left(1_{0}-0_{0}\right)=A_{u l} / \gamma_{u l}$, is, thus, $\sim 3 \times 10^{7} \mathrm{~cm}^{-3}$, which is, as in the case of the $\mathrm{H}_{2} \mathrm{O}$ line discussed above, many orders of magnitude higher than the densities (of order a few times $10^{3} \mathrm{~cm}^{-1}$ ) of the absorbing interstellar medium (ISM) in PKS 1830-211. It is therefore also clear that the population of the $\mathrm{NH}_{3} 1_{0}$ relative to the $0_{0}$ level is controlled by the cosmic microwave background temperature. Using Eq. (3), we calculate $N_{1_{0}}=1.4 \times 10^{12} \mathrm{~cm}^{-2}$ for the column density of the $1_{0}$ level. We can calculate the ortho- $\mathrm{NH}_{3}$ partition function by direct summation with parameters from LAMDA and get, assuming $T_{\text {rot }}=5.145 \mathrm{~K}, Q_{\mathrm{o}-\mathrm{NH}_{3}}(5.145 \mathrm{~K}) \approx 4.06$. Using Eq. (4) we find that the total ortho- $\mathrm{NH}_{3}$ column density, $\mathrm{N}\left(\mathrm{o}-\mathrm{NH}_{3}\right)$, is $9.1 \times 10^{13} \mathrm{~cm}^{-2}, 70$ times higher than $N_{10}$.

Again using RADEX, we find that to reproduce our observed opacity $\left(0.62\right.$ at a line width of $\left.34 \mathrm{~km} \mathrm{~s}^{-1}\right)$ requires an orthoammonia column density, $N\left(\mathrm{o}-\mathrm{NH}_{3}\right)$, of $1.3 \times 10^{14} \mathrm{~cm}^{-2}$. For $T_{\text {rot }}=5.145 \mathrm{~K}$, a quarter of all ortho- $\mathrm{NH}_{3}$ molecules are in the $0_{0}$ ground-state level while, in contrast to $\mathrm{o}-\mathrm{H}_{2} \mathrm{O}$, the majority of molecules are in other energy levels. We used a kinetic temperature of $80 \mathrm{~K}$ (see Sect. 1 and Henkel et al. 2008) but note that lower temperatures would only imply a $20 \%$ lower column density at the most.

\section{Discussion - Abundances and astrochemical implications}

To calculate molecular abundances relative to molecular hydrogen, we assume an $\mathrm{H}_{2}$ column density of $3 \times 10^{22} \mathrm{~cm}^{-1}$ for the absorbing material toward PKS 1830-211-SW. This value was estimated by Wiklind \& Combes (1996) assuming a $\mathrm{CO}$ to $\mathrm{H}_{2}$ ratio of $10^{-4}$. It is somewhat larger than the $(1.8 \pm 0.3) \times 10^{22} \mathrm{~cm}^{-2} \mathrm{de}-$ rived from X-ray observations with the ROSAT satellite (Mathur \& Nair 1997).

With the lower limit to $N\left(\mathrm{o}-\mathrm{H}_{2} \mathrm{O}\right)$ determined above, $1.2 \times$ $10^{14} \mathrm{~cm}^{-2}$, we find that the ortho- $\mathrm{H}_{2} \mathrm{O}$ to $\mathrm{H}_{2}$ abundance ratio, $X\left(\mathrm{o}-\mathrm{H}_{2} \mathrm{O}\right)$, is greater than $\approx 5 \times 10^{-9}$. To put this value into context, SWAS observed the same $\mathrm{H}_{2} \mathrm{O}$ line in a wide variety of Galactic sources (for an overview see Melnick et al. 2000). Snell et al. (2000a) were able to determine $\mathrm{H}_{2} \mathrm{O}$ abundances, with values similar to our PKS 1830-211 number, toward dense quiescent regions of molecular clouds (between $6 \times 10^{-10}$ and $1 \times 10^{-8}$ ), with values near the higher number found in the core regions of Giant Molecular Clouds (GMCs, see also Bergin et al. 2000).

Several observational effects have to be considered concerning the SWAS results: the regions toward which SWAS detected the $\mathrm{H}_{2} \mathrm{O}$ line at low abundance are core regions of molecular clouds, some of them GMCs, i.e., regions of enhanced density and thus, enhanced column density, producing high enough optical depths to make the line detectable. They are quiescent in the sense they do not harbor outflows. Whereas toward the spatially confined regions harboring outflows, as indicated by broad line wings and other tracers, SWAS finds several orders of magnitude higher $\mathrm{H}_{2} \mathrm{O}$ abundances (Bergin et al. 2000, 2003). Similarly, higher $\mathrm{H}_{2} \mathrm{O}$ abundances are found near the warmer interfaces to HII regions (Snell et al. 2000b). SWAS gives little information, just rough upper limits, on water in the lower density extended gas of giant molecular clouds, simply because the expected line intensities are prohibitively low. Similarly, only coarse upper limits (around $10^{-7}-10^{-6}$ relative to $\mathrm{H}_{2}$ ) can be derived toward cold dark clouds such as TMC-1, L134N, or B335, which also have lower average densities $\left(10^{4} \mathrm{~cm}^{-4}\right)$ than warm GMC cores. Moreover, their low temperatures $(10 \mathrm{~K})$ are not 
conducive for producing emission in the $1_{10}-1_{01}$ line, whose upper energy level is $34 \mathrm{~K}$ above ground.

The size of the $\sim 4^{\prime} F W H M$ beam of SWAS at the $\mathrm{H}_{2} \mathrm{O}$ line rest frequency $(557 \mathrm{GHz})$ for a region at distance $D$ (measured in $\mathrm{kpc}$ ) corresponds to an area of size $\approx 1 \mathrm{pc} \times D(\mathrm{kpc})$. This means that for sources at a few kpc distance, similar size scales are sampled as for PKS 1830-211 with the VLBA beam of Carilli et al. (1998), which has a $F W H M$ of 1 mas $(7.5$ pc at $z=0.89)$. Indeed, the SWAS $\mathrm{H}_{2} \mathrm{O}$ spectrum of the $\mathrm{W} 33$ region, which is at a (near-kinematic) distance of $4 \mathrm{kpc}$, has a width of $\sim 25 \mathrm{~km} \mathrm{~s}^{-1}$ full width at zero power $(\mathrm{FWZP})^{5}$. This is very wide for a quiescent cloud core which, as the low $\mathrm{H}_{2} \mathrm{O}$ abundance suggests, does not contain outflows. The width could be determined by a combination of the presence of velocity gradients in the region unresolved by the beam and the existence of emission from several spiral arms caused by the line of sight crossing the plane of the Galaxy. Alternately, an outflow could be present, which actually is suggested by emission from the outflow tracer $\mathrm{SiO}$ from which lines of width comparable to the $\mathrm{H}_{2} \mathrm{O}$ line's are observed toward W33 (Miettinen et al. 2006). In that case, the apparent low $\mathrm{H}_{2} \mathrm{O}$ abundance could result from the superposition of a compact (i.e. beam diluted) higher-abundance outflow component and a more extended component of much lower abundance.

Even in comparison to the relatively broad $\mathrm{H}_{2} \mathrm{O}$ emission toward W33, the 55-60 $\mathrm{km} \mathrm{s}^{-1}$ FWZP width of the PKS 1830-211-SW absorption appears extraordinarily broad, especially since we are viewing the lensing galaxy close to faceon and the 2 milliarcsec size absorption "pencil beam" afforded by the size of the background source corresponds to a scale of $15 \mathrm{pc}$, smaller than a single GMC. Interferometric observations of the local face-on spiral M 51 with a resolution corresponding to a much larger $\sim 150 \mathrm{pc}$ reveal linewidths only between 10 and $20 \mathrm{~km} \mathrm{~s}^{-1}$ in the galaxy's spiral arms (Aalto et al. 1999).

The probability of a random line of sight passing through a cloud core certainly is lower than passing through a lower density, more "normal", region of a GMC. The $\mathrm{H}_{2} \mathrm{O}$ abundance in the bulk volume of molecular clouds is essentially unknown and, for the above reasons, SWAS or ODIN cannot deliver very meaningful limits. As the multi-molecule studies of galactic absorption against extragalactic radio sources conducted by Liszt and Lucas have persuasively demonstrated, all the other molecules found toward PKS 1830-211 can be detected in general molecular ISM gas (Lucas \& Liszt 2000; Liszt \& Lucas 2001; Liszt et al. 2004; Liszt \& Lucas 2004; Liszt et al. 2006). However, as explained in the following, the gas absorbing PKS 1830-211 appears to have a much higher temperature.

Using the NRAO Green Bank Telescope, Henkel et al. (2008) detected absorption in the $\mathrm{NH}_{3}(J, K)$ inversion lines toward PKS 1830-211 for $J=K=1$ up to 10 , which arise from levels with energies between 23 and $1036 \mathrm{~K}$ above ground. Figure 2 compares our spectrum of the $J_{K}=1_{1}-0_{0}$ line with their $J, K=(1,1)$ spectrum. There appears to be excess absorption in the former at velocities $>0 \mathrm{~km} \mathrm{~s}^{-1}$, although the signalto-noise ratio is rather limited. If real, this would be difficult to explain since, given that the absorbed region is larger at the redshifted $(1,1)$ frequency $(12.56 \mathrm{GHz})$ than at the $1_{1}-0_{0}$ frequency, one would expect the opposite. Higher signal to noise data obtainable with the Submillimeter Array seem highly desirable!

Henkel et al. (2008) find that to explain the observed inversion line optical depths, $80-90 \%$ of the $\mathrm{NH}_{3}$ must be in warm

\footnotetext{
5 Intriguingly, the W33 spectrum shows absorption against the source's strong submillimeter continuum emission in its central velocity range.
}

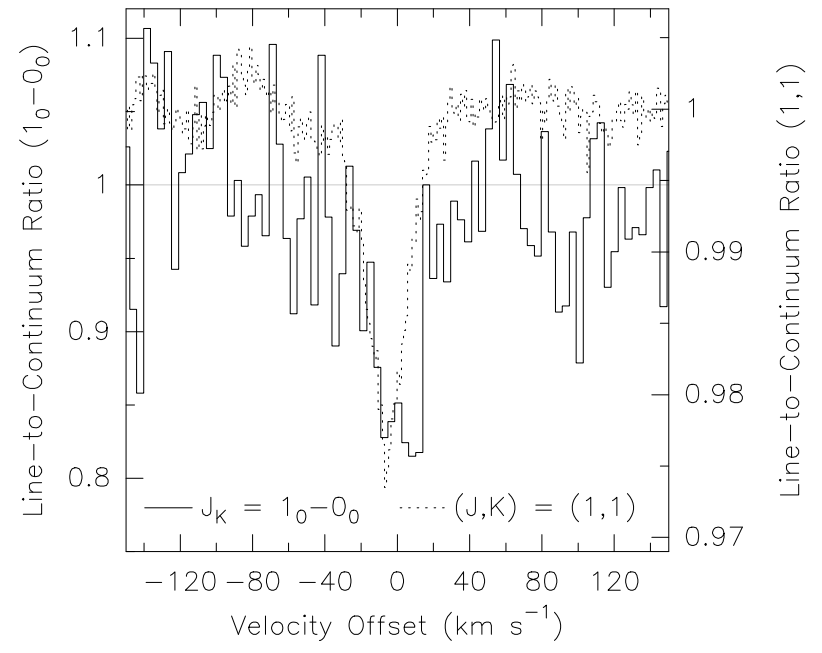

Fig. 2. Ammonia absorption spectra of the $J_{K}=1_{0}-0_{0}$ rotational transition (full line) and the $(J, K)=(1,1)$ inversion transition (dashed line) toward PKS 1830-211. The velocity scale is in the heliocentric frame and assumes a redshift of 0.88582. The $y$-axes give the line-tocontinuum ratio for the $1_{0}-0_{0}$ line (left), for which the continuum level is $1.65 \mathrm{Jy}$, and the $(1,1)$ line (right) for which the continuum level is $8.2 \mathrm{Jy}$.

$(80 \mathrm{~K})$ gas with a total ortho- plus para- $\mathrm{NH}_{3}$ column density of $5 \times 10^{14} \mathrm{~cm}^{-2}$, if the excitation temperature (assumed to be the same for all lines) equals $T_{\mathrm{CMB}}$. When modeling the clouds in their SWAS sample, Snell et al. (2000b) assumed temperatures between 25 and $40 \mathrm{~K}$ for the GMC cores, as determined from observations of other tracers, which is significantly lower than the $80 \mathrm{~K}$ quoted above. Moreover, the kinetic temperatures of 25$30 \mathrm{~K}$ derived by Liszt et al. (2006) from the $\mathrm{NH}_{3}(1,1)$ and $(2,2)$ lines are substantially lower. This poses an interesting question: is the $z=0.89$ absorbing cloud much hotter than Galactic clouds or could a dilute warm molecular component in the Milky Way (and other galaxies) so far have escaped observational attention?

The ortho- $\mathrm{NH}_{3}$ column density determined by us is a factor of 2 lower than the value derived from inversion lines (Henkel et al. 2008). Interestingly, a much larger discrepancy seems to exist between the $\mathrm{NH}_{3}$ column density derived from the Odin $1_{1}-0_{0}$ spectrum (Liseau et al. 2003) and the much higher value implied by the $(1,1)$ and $(2,2)$ inversion lines mapped by Zeng et al. (1984) toward the dark cloud $\rho$ Oph A.

\section{Outlook - constraints on variation of fundamental constants}

Comparing the redshifts of various spectral lines measured toward the same high- $z$ object can in principle yield constraints on possible temporal variations of fundamental constants over significant parts of the age of the Universe (see, e.g. Curran et al. 2004). A precondition is that the lines in question have different dependencies on the constants, e.g., the fine structure constant, $\alpha$, and/or the ratio of the mass of the proton to that of the electron, $\mu=m_{\mathrm{p}} / m_{\mathrm{e}}$. Traditionally exploited with optical/UV quasar absorption lines, the method has recently been applied to radio and millimeter absorption lines toward, amongst others, the gravitational lens systems PMN J0134-0931 (Kanekar et al. 2005) and B0218+357 (Murphy et al. 2008).

The ammonia molecule is especially interesting with respect to this, as its inversion transitions are strongly dependent on $\mu$ : Flambaum \& Kozlov (2007) find that the fractional change in 
frequency of these transitions $\delta v_{\text {inv }} / v_{\text {inv }}=\delta z /(1+z)$ is given by $-4.46 \delta \mu / \mu$ or, in other words, $\Delta v_{\text {inv }} \propto \Delta \mu^{-4.46}$. For fractional variations of rotational line frequencies, $v_{\text {rot }}$, in contrast, the proportionality factor is unity, i.e., $\delta v_{\text {rot }} / \nu_{\text {rot }}=-\delta \mu / \mu$. Comparing $\mathrm{NH}_{3}$ inversion line redshifts with redshifts of rotational lines should thus provide sensitive limits on the variation of $\mu$.

Murphy et al. (2008) compare the measured redshifts of the $\mathrm{NH}_{3}(J, K)=(1,1),(2,2)$ and $(3,3)$ lines observed toward B0218+357 by Henkel et al. (2005) with rotational lines from $\mathrm{HCO}^{+}$and $\mathrm{HCN}$ and obtain $\delta \mu / \mu=(0.74 \pm 0.47) \times 10^{-6}$ over a look-back time of $6.2 \mathrm{Gyr}$, corresponding to the absorber's redshift of 0.68466 . One fundamental limitation of this measurement is the uncertainty over the two species' possibly different distributions leading to different covering factors. Different covering factors might also result from source size variations between $14 \mathrm{GHz}\left(\mathrm{NH}_{3}\right)$ and $105 \mathrm{GHz}\left(\mathrm{HCO}^{+}\right.$and $\left.\mathrm{HCN}\right)$. In addition, lines from different species might also cover different velocity ranges.

The ortho-ground-state rotational transition of $\mathrm{NH}_{3}$ we have detected toward PKS 1830-211 can in principle deliver a more accurate and meaningful comparison between the redshifts of a rotational line redshift and of the inversion lines, which have been determined by Henkel et al. (2008, see Sect. 1). The velocity accuracy of our APEX measurement $\left(2.5 \mathrm{~km} \mathrm{~s}^{-1}\right)$ translates into a redshift uncertainty, $\delta z$, of $1.6 \times 10^{-5}$ and a value of $\delta \mu / \mu \approx 0.224 \delta z /(1+z)$ of $1.9 \times 10^{-6}$, which is three times larger than the value derived by Murphy et al. (2008) for B0218+357.

Our $\mathrm{NH}_{3} 1_{0}-0_{0}$ spectrum is of very limited quality and even looks quite different from the $(1,1)$ spectrum (see Fig. 2), although that can be attributed to the former line's poor signal-tonoise ratio. A few hour long observation with the Submillimeter Array (Ho et al. 2004) will result in a much improved spectrum, which, together with the $(1,1)$ spectrum could lead to a significant advancement in investigating the variation of $\mu$ over the past 7.2 Gyr.

Acknowledgements. We would like to thank Gary Melnick for useful comments on this paper.

\section{References}

Aalto, S., Hüttemeister, S., Scoville, N. Z., \& Thaddeus, P. 1999, ApJ, 522, 165 Bergin, E. A., Melnick, G. J., Stauffer, J. R., et al. 2000, ApJ, 539, L129

Bergin, E. A., Kaufman, M. J., Melnick, G. J., Snell, R. L., \& Howe, J. E. 2003, ApJ, 582, 830

Bronfman, L., Cohen, R. S., Alvarez, H., May, J., \& Thaddeus, P. 1988, ApJ, 324,248

Carilli, C. L., Menten, K. M., Reid, M. J., Rupen, M., \& Claussen, M. 1998, in Radio Emission from Galactic and Extragalactic Compact Sources, ed. J. A. Zensus, G. B. Taylor, \& J. M. Wrobel, IAU Colloq., 164, ASP Conf. Ser., 144,317

Chengalur, J. N., de Bruyn, A. G., \& Narasimha, D. 1999, A\&A, 343, L79
Combes, F., \& Wiklind, T. 1997, ApJ, 486, L79

Courbin, F., Meylan, G., Kneib, J.-P., \& Lidman, C. 2002, ApJ, 575, 95

Curran, S. J., Kanekar, N., \& Darling, J. K. 2004, New Astron. Rev., 48, 1095

Danby, G., Flower, D. R., Valiron, P., Schilke, P., \& Walmsley, C. M. 1988, MNRAS, 235, 229

Flambaum, V. V., \& Kozlov, M. G. 2007, Phys. Rev. Lett., 98, 240801

Frye, B., Welch, W. J., \& Broadhurst, T. 1997, ApJ, 478, L25

Grosjean, A., Dubernet, M.-L., \& Ceccarelli, C. 2003, A\&A, 408, 1197

Güsten, R., Nyman, L. Å., Schilke, P., et al. 2006, A\&A, 454, L13

Henkel, C., Jethava, N., Kraus, A., et al. 2005, A\&A, 440, 893

Henkel, C., Braatz, J. A., Menten, K. M., \& Ott, J. 2008, A\&A, 485, 451

Hjalmarson, Å., Frisk, U., Olberg, M., et al. 2003, A\&A, 402, L39

Ho, P. T. P., Moran, J. M., \& Lo, K. Y. 2004, ApJ, 616, L1

Huettemeister, S., Wilson, T. L., Mauersberger, R., et al. 1995, A\&A, 294, 667

Jauncey, D. L., Reynolds, J. E., Tzioumis, A. K., et al. 1991, Nature, 352, 132

Jin, C., Garrett, M. A., Nair, S., et al. 2003, MNRAS, 340, 1309

Kanekar, N., Carilli, C. L., Langston, G. I., et al. 2005, Phys. Rev. Lett., 95, 261301

Klein, B., Philipp, S. D., Krämer, I., et al. 2006, A\&A, 454, L29

Koopmans, L. V. E., \& de Bruyn, A. G. 2005, MNRAS, 360, L6

Lidman, C., Courbin, F., Meylan, G., et al. 1999, ApJ, 514, L57

Liseau, R., Larsson, B., Brandeker, A., et al. 2003, A\&A, 402, L73

Liszt, H., \& Lucas, R. 2001, A\&A, 370, 576

Liszt, H., \& Lucas, R. 2004, A\&A, 428, 445

Liszt, H., Lucas, R., \& Black, J. H. 2004, A\&A, 428, 117

Liszt, H. S., Lucas, R., \& Pety, J. 2006, A\&A, 448, 253

Lovell, J. E. J., Reynolds, J. E., Jauncey, D. L., et al. 1996, ApJ, 472, L5

Lovell, J. E. J., Jauncey, D. L., Reynolds, J. E., et al. 1998, ApJ, 508, L51

Lucas, R., \& Liszt, H. S. 2000, A\&A, 358, 1069

Mathur, S., \& Nair, S. 1997, ApJ, 484, 140

Melnick, G. J., Stauffer, J. R., Ashby, M. L. N., et al. 2000, ApJ, 539, L77

Menten, K. M., Carilli, C. L., \& Reid, M. J. 1999, in Highly Redshifted Radio Lines, ed. C. L. Carilli, S. J. E. Radford, K. M. Menten, \& G. I. Langston, ASP Conf. Ser., 156, 218

Meylan, G., Courbin, F., Lidman, C., Kneib, J.-P., \& Tacconi-Garman, L. E. 2005, A\&A, 438, L37

Miettinen, O., Harju, J., Haikala, L. K., \& Pomrén, C. 2006, A\&A, 460, 721

Muller, S., Guélin, M., Dumke, M., Lucas, R., \& Combes, F. 2006, A\&A, 458, 417

Murphy, M. T., Flambaum, V. V., Muller, S., \& Henkel, C. 2008, Science, 320, 1611

Nordh, H. L., von Schéele, F., Frisk, U., et al. 2003, A\&A, 402, L21

Phillips, T. R., Maluendes, S., \& Green, S. 1996, ApJS, 107, 467

Risacher, C., Vassilev, V., Monje, R., et al. 2006, A\&A, 454, L17

Schöier, F. L., van der Tak, F. F. S., van Dishoeck, E. F., \& Black, J. H. 2005, A\&A, 432, 369

Snell, R. L., Howe, J. E., Ashby, M. L. N., et al. 2000a, ApJ, 539, L97

Snell, R. L., Howe, J. E., Ashby, M. L. N., et al. 2000b, ApJ, 539, L101

Spergel, D. N., Bean, R., Doré, O., et al. 2007, ApJS, 170, 377

Subrahmanyan, R., Narasimha, D., Pramesh-Rao, A., \& Swarup, G. 1990, MNRAS, 246, 263

Subrahmanyan, R., Kesteven, M. J., \& Te Lintel Hekkert, P. 1992, MNRAS, 259, 63

van der Tak, F. F. S., Black, J. H., Schöier, F. L., Jansen, D. J., \& van Dishoeck, E. F. 2007, A\&A, 468, 627

Wiklind, T., \& Combes, F. 1996, Nature, 379, 139

Wiklind, T., \& Combes, F. 1998, ApJ, 500, 129

Wiklind, T., \& Combes, F. 2005, in BAAS, 37, 1424

Wilson, T. L., Henkel, C., \& Hüttemeister, S. 2006, A\&A, 460, 533

Winn, J. N., Kochanek, C. S., McLeod, B. A., et al. 2002, ApJ, 575, 103

Zeng, Q., Batrla, W., \& Wilson, T. L. 1984, A\&A, 141, 127 